\title{
STATISTICAL ANALYSIS OF 26 YEARS OF OBSERVATIONS OF DECAMETRIC RADIO EMISSIONS FROM JUPITER
}

\author{
M. S. Marques*†, P. Zarka ${ }^{\dagger}$ E. Echer*, V. B. Ryabov ${ }^{\ddagger}$, and M. V. Alves*
}

\begin{abstract}
Jupiter appears to be a complex radio source in the decameter wavelength range. As a consequence, statistical studies based on long-term observations are required in order to understand the various phenomena observed. We present a statistical analysis of 26 years of observations, in digital format, recorded by the Nançay Decameter Array, France. The emissions were classified using arc shape (in the timefrequency plane), maximum event frequency, and dominant polarization, with the aim of distinguishing several types of emissions (Io or non-Io, northern or southern, dawn or dusk). The results are generally consistent with previous studies. However, the large time coverage of this new database, together with the new classification method adopted and the better precision in identifying emissions contours due to the digital data format, allowed us to identify new types of emissions (Io-A", Io-B' and non-Io-D), as well as changes on the limits of emission regions in the CMLIo phase diagram. Another significant result is the new, better defined maximum frequency envelopes of the emissions, especially Io-C, Io-D (as a function of Io's longitude) and non-Io-C emissions. Finally, we will present preliminary results on long-term variations of the emissions.
\end{abstract}

\footnotetext{
* National Institute for Space Research (INPE), Sao Jose dos Campos, Brazil

$\dagger$ LESIA \& USN Observatoire de Paris/CNRS/PSL, Meudon, France

$\ddagger$ Complex Systems Department, Future University Hakodate, Hakodate, Japan
} 
